Western University

Scholarship@Western

Chemistry Publications

Chemistry Department

$12-5-2017$

\title{
1,6-Diphenyl-1,3,5-hexatriene (DPH) as a Novel Matrix for MALDI MS Imaging of Fatty Acids, Phospholipids, and Sulfatides in Brain Tissues.
}

Hanadi Ibrahim

Kristina Jurcic

Jasmine S-H Wang

Shawn N Whitehead

Ken K-C Yeung

kyeung@uwo.ca

Follow this and additional works at: https://ir.lib.uwo.ca/chempub

Part of the Chemistry Commons

Citation of this paper:

Ibrahim, Hanadi; Jurcic, Kristina; Wang, Jasmine S-H; Whitehead, Shawn N; and Yeung, Ken K-C, "1,6-Diphenyl-1,3,5-hexatriene (DPH) as a Novel Matrix for MALDI MS Imaging of Fatty Acids, Phospholipids, and Sulfatides in Brain Tissues." (2017). Chemistry Publications. 82.

https://ir.lib.uwo.ca/chempub/82 


\section{1,6-Diphenyl-1,3,5-hexatriene (DPH) as a Novel Matrix for MALDI MS Imaging of Fatty Acids,}

Phospholipids, and Sulfatides in Brain Tissues

Hanadi Ibrahim ${ }^{1}$, Kristina Jurcic ${ }^{2}$, Jasmine S.-H. Wang ${ }^{1}$, Shawn N. Whitehead ${ }^{3}$ and Ken K.-C. Yeung ${ }^{1,2^{*}}$

${ }^{1}$ Department of Chemistry, ${ }^{2}$ Department of Biochemistry and ${ }^{3}$ Department of Anatomy and Cell Biology, The University of Western Ontario, London, ON, Canada

This article was also published in Analytical Chemistry (Anal. Chem. 89, 23, 12828-12836)

\section{Abstract}

1,6-Diphenyl-1,3,5-hexatriene (DPH) is a commonly used fluorescence probe for studying cell membranelipids due to its affinity toward the acyl chains in the phospholipid bilayers. In this work, we investigated its use in matrix-assisted laser desorption/ionization (MALDI) as a new matrix for mass spectrometry imaging (MSI) of mouse and rat brain tissue. DPH exhibits very minimal matrix-induced background signals for the analysis of small molecules (below $\mathrm{m} / \mathrm{z}$ of 1000). In the negative ion mode, DPH permits the highly sensitive detection of small fatty acids (m/z $200-350)$ as well as a variety of large lipids up to $\mathrm{m} / \mathrm{z}$ of 1000, including lyso-phospholipid, phosphatidic acid (PA), phosphoethanolamine (PE), phosphatidylserine (PS), phosphatidylglycerol (PG), phosphatidylinositol (PI), and sulfatides (ST). The analytes were mostly detected as the deprotonated ion $[\mathrm{M}-\mathrm{H}]^{-}$. Our results also demonstrate that sublimated DPH is stable for at least $24 \mathrm{~h}$ under the vacuum of our MALDI mass spectrometer. The ability to apply DPH via sublimation coupled with its low volatility allows us to perform tissue imaging of the above analytes at high spatial resolution. The degree of lipid fragmentation was determined experimentally at varying laser intensities. The results illustrated that the use of relatively low laser energy is important to minimize the artificially generated fatty acid signals. On the other hand, the lipid fragmentation obtained at higher laser energies provided tandem MS information useful for lipid structure elucidation.

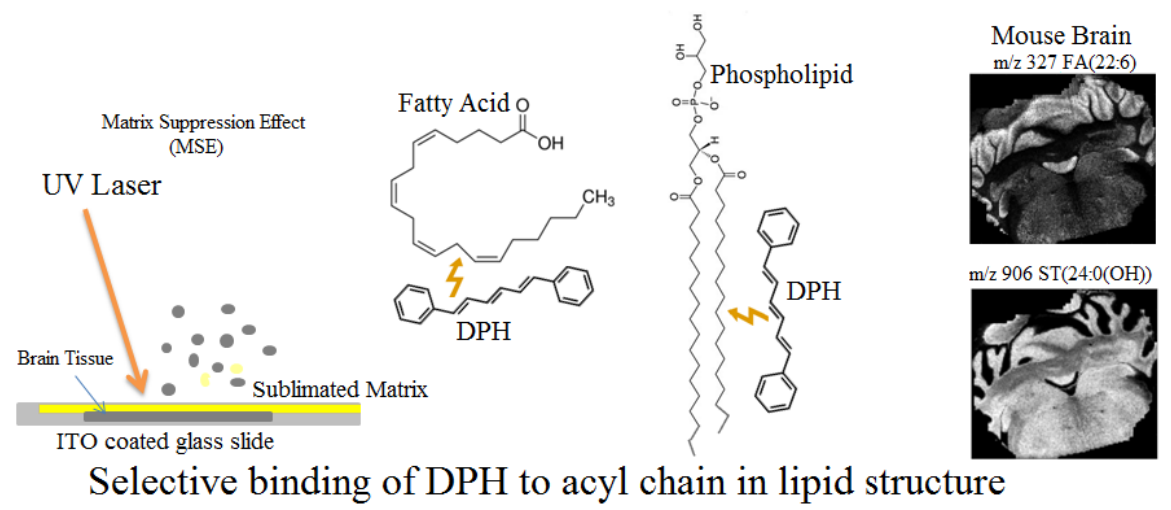

*Corresponding author Email: kyeung@uwo.ca 


\section{Introduction}

During the MALDI process, ${ }^{1}$ the matrix can play multiple roles including the mediator of energy transfer, the proton donor and/or receptor and the analyte selector that gives rise to selectivity. Continuous effort in exploring new matrices is desirable, not only to improve the quality of MALDI MS data based on the analyte selectivity/specificity of the matrices ${ }^{2}$, but also to gain a better understanding of the analyte desorption/ionization mechanism and analyte-matrix interactions. ${ }^{3}$ Generally, examples of ideal characteristics of MALDI matrices may include: a strong absorption at the wavelength of the irradiating laser, ${ }^{4}$ minimal background signals originated from the matrix and its clusters, the ability to co-crystallize with the analyte and promote its ionization, negligible volatility at room temperature and high stability under the vacuum of mass spectrometers. For MS imaging, it is also desirable for the matrix to be compatible with application by sublimation, which produces the highly homogenous matrix layer required for high spatial resolution. ${ }^{5}$

In the matrix-analyte cocrystallization step, it has been proven that thorough mixing and integration between the matrix and the analyte molecules ${ }^{6}$ is crucial for obtaining both the optimal ionization of analytes $^{7}$ and the suppression of background matrix ion signals. ${ }^{8}$ In other words, it is ideal to select a matrix with high chemical affinity and compatibility with the analytes of interest. For example, Fukuyama et al. synthesized a novel matrix additive o-alkylated dihydroxybenzoic acid (ADHB) to increase the hydrophobicity of the conventional 2,5-dihydroxybenzoic acid (DHB) and, thus, enhancing its affinity toward hydrophobic peptides and improve the sensitivity. ${ }^{9}$ The authors claimed that ADHB enriches the hydrophobic part and this enrichment depends on the degree of hydrophobicity. Similarly, Stoyanovsky and co-workers investigated the application of DHB- $\mathrm{C}_{n} \mathrm{H}_{2 \mathrm{n}+1}\left(\mathrm{C}_{6} \mathrm{H}_{13}, \mathrm{C}_{9} \mathrm{H}_{19}\right.$ and $\left.\mathrm{C}_{12} \mathrm{H}_{25}\right)$ for MALDI-MSI of phospholipids in brain tissue. ${ }^{10}$ Comparing to the conventional DHB, the modified DHB promoted the formation of finer crystals through enhanced incorporation of the alkyl chains of $\mathrm{DHB}-\mathrm{C}_{\mathrm{n}} \mathrm{H}_{2 \mathrm{n}+1}$ into hydrophobic phospholipid bilayers and resulted in improved spatial resolution.

Various phospholipids and fatty acids ${ }^{11}$ have been identified as biomarkers of diseases such as Alzheimer's disease, ${ }^{12}$ schizophrenia, ${ }^{13}$ coronary heart diseases, ${ }^{14}$ cancers ${ }^{15}$ and lacunar infarction. ${ }^{16}$ The development in biomolecular imaging techniques enabled the revelation of spatial distribution of several lipids ${ }^{17}$ and better understanding of their role in biological processes ${ }^{18}$ and diseases. ${ }^{19} \mathrm{DHB},{ }^{17,20}$ 9-aminoacridine (9$\mathrm{AA})^{17,21}$ and 1,5-diaminonaphtalene (DAN) ${ }^{22}$ are the most widely used matrices for lipid MS imaging. However, the MS imaging of fatty acids is more difficult owing to the following two challenges. First, common matrices do not sufficiently facilitate the detection fatty acids via MALDI due to the intense matrix-induced background signals at the same MW region as that of fatty acids and/or the poor ionization 
efficiency of fatty acid molecules. 1,8-Bis(dimethylamino) naphthalene (DMAN) was reported as a "proton sponge", a super basic matrix suitable for MALDI MS analysis of metabolites, ${ }^{23}$ fatty acids ${ }^{24}$ and other lipids. ${ }^{25}$ However, DMAN is not stable under high vacuum. That is, the deposited matrix is sufficiently volatile at ambient temperature for it to sublime under the MALDI MS vacuum. DMAN coatings on sample targets were reported to disappear completely after $60 \mathrm{~min}$ and thus cannot be used for imaging experiments which are often longer than $1 \mathrm{~h} .{ }^{22}$ Alternative to matrices of organic molecules, the use of metallic silver, either as nanoparticle ${ }^{26,27}$ or metallic sputtered films ${ }^{28,29}$, was found effective for the laser desorption/ionization (LDI) MS imaging of biological tissues. In particular, Hayasaka et al. ${ }^{27}$ reported the use of silver nanoparticles for the visualization of fatty acids. Instead of discovering novel matrices, another approach was to perform on-tissue chemical derivatization of fatty acids, with the intentions of enhancing ionization efficiency and shifting the molecular mass of fatty acids away from the matrix background. ${ }^{30}$

The second and more problematic challenge is the inability to distinguish the signals from endogenous free fatty acids in the tissue than those resulted from degradation or dissociation of lipids. Specifically, the authors of both refs ${ }^{27}$ and ${ }^{30}$ acknowledged that the MS signals of fatty acids in their reports could come from either: the desired free fatty acids present in the samples, or the undesired fragments dissociated from lipids either in gas phase during ionization/desorption or on-tissue during sample preparation and/or matrix application. To minimize this fragmentation, $\mathrm{Wu}$ and co-workers published the use of dopamine-modified $\mathrm{TiO}_{2}$ monolith for LDI MS imaging of small metabolites, fatty acids and lipids. Using dopamine as a ligand allowed them to shift the UV absorption of $\mathrm{TiO}_{2}$ toward the laser emission wavelength and increase the sensitivity and to increase the $\mathrm{pH}$ of the tissue surface and minimize the hydrolysis of lipids. ${ }^{31}$

In this paper, we propose the use of commercially available 1,6-diphenyl-1,3,5-hexatriene (DPH) as a novel matrix for MALDI MS imaging analysis of a variety of fatty acids and lipids, without the need of any chemical derivatization. DPH is a polyene hydrocarbon compound and is one of the most widely used fluorescent probes in studies of biological membranes ${ }^{32}$ and phospholipids bilayer. ${ }^{33}$ Due to the continuous pi bonds $\left(\mathrm{C}_{6} \mathrm{H}_{5}-\mathrm{CH}=\mathrm{CH}-\mathrm{CH}=\mathrm{CH}-\mathrm{CH}=\mathrm{CH}-\mathrm{C}_{6} \mathrm{H}_{5}\right)$, DPH has three vibrational peaks, its absorption band ranges from 330-380 nm, ${ }^{34}$ which matches the emission wavelengths of common MALDI lasers. Consisting of only hydrocarbons in a nonpolar configuration, DPH exhibits high hydrophobic affinity toward the alkyl chains in the lipids, ${ }^{35}$ which is supported by the well-documented DPH integration within cell membranes. ${ }^{36}$ DPH was also generally used in lipid detection for its emission enhancement in the presence of phospholipids and triglycerides compounds in aqueous media. ${ }^{37}$ In addition, it was shown that DPH can transfer its excitation energy to nearby acceptor molecule (chromophore). ${ }^{38}$ All the above criteria support DPH being an excellent candidate as a matrix for the detection of fatty acids and phospholipids by MALDIMS. To the best of our knowledge, this is the first report of applying DPH as a matrix for MALDI MS and 
MSI analyses. Experiments will be presented in this paper to demonstrate that DPH is a suitable and effective matrix to facilitate the MALDI MS imaging of lipids in rat and mouse brain tissues, including a variety of lipids: fatty acids (FAs), lyso-phospholipids (L-PLs), phospholipids (PLs), and sulfatides (STs). The degree of lipid fragmentation with DPH as matrix will also be investigated for its application in the MALDI MS tissue imaging of free fatty acids. 


\section{Materials and Methods}

Chemicals. Liquid chromatography grade solvents, methanol, ethanol, chloroform, and tetrahydrofuran (THF), were purchased from Sigma-Aldrich (Oakville, ON, Canada). Various matrices, 9-aminoacridine

(9-AA), 1,5-diaminonaphthalene (DAN), 2,5 dihydroxybenzoic acid (DHB), 1,8bis(dimethylamino)naphthalene (DMAN), $\alpha$-cyano-4-hydroxycinnamic acid (CHCA), 1,6-diphenyl-1,3,5hexatriene (DPH), and fatty acid standards, hexanoic, palmitic, and arachidic acids, were also purchased from Sigma-Aldrich. DPH was recrystallized using acetone prior to use. Briefly, $5 \mathrm{~g}$ of DPH were dissolved in about $125 \mathrm{~mL}$ of acetone at its boiling point $\left(56^{\circ} \mathrm{C}\right)$. The hot solution was filtered to remove insoluble impurities. Crystals were formed as the solution cooled to room temperature. They were isolated by filtration and dried.

Lipid standards were purchased from Avanti Polar Lipids, Inc. (Alabaster, AL, USA), including, 1,2dilauroyl-sn-glycero-3-phosphocholine (DLPC), 1,2-dimyristoyl-sn-glycero-3-phosphate (DMPA, as sodium salt), 1-palmitoyl-2-hydroxy-sn-Glycero-3-phosphate (lyso-PA 16:0), 1-(1z-hexdecyl)-sn-glycero3-phosphocholine (lyso-PC 16:1), 1,2-distearoyl-sn-Glycero-3-Phosphoethanolamine (DSPE), 1,2distearoyl-sn-glycero-3-[Phospho-rac-(1-glycerol)] (DSPG, as sodium salt) and 1,2-dioleoyl-sn-glycero-3[phospho-L-serine] (DOPS, as sodium salt).

Stock solutions were prepared at a concentration of $0.5 \mathrm{mg} / \mathrm{mL}$ in various solvent mixtures: ethanol for fatty acids, methanol for lyso-PA, DMPA and DLPC, a methanol-to-chloroform of 1:1 for DSPG, DSPE and DOPS, and chloroform for lyso-PC. Solutions of DPH were prepared in THF. Solutions of 9-AA, CHCA, DAN, DHB and DMAN were prepared in methanol.

Sample Preparation for Standards. Dried droplet preparations were performed; equal volumes from analyte and matrix solutions are mixed, then $0.75 \mu \mathrm{L}$ was deposited on the target and allowed to dry. For individual standards analysis, the deposited amount of analyte is $0.5 \mathrm{nmol}$. For the analyses of phospholipid mixture standards, in comparing the effect of organic matrices, the deposited amount of each phospholipid was 50 pmol.

Tissue Sample Preparation. Brains from male of C57BL/6 mice and Wistar rats (aged 6 months) were used for tissue sections. The brains were flash-frozen in dry ice immediately after dissection and stored at $-80{ }^{\circ} \mathrm{C}$ until use. They were attached to a specimen holder on dry ice with distilled water. Using a cryostat (CryoStar NX50, ThermoFisher Scientific, Burlington, ON, Canada), $10 \mu \mathrm{m}$ thick coronal and sagittal brain sections were thaw-mounted onto indium tin oxide coated glass slides (Hudson Surface Technology Inc., 
Fort Lee, NY, USA). Slides were dehydrated for 5-10 min in a vacuum chamber and then coated with DPH matrix using a sublimation apparatus (Chemglass Life Sciences, Vineland NJ, USA). Following matrix application, a mixture of lyso-PA and the calibration standard kit (SCIEX, Concord, ON, Canada) was mixed with $\mathrm{CHCA}$, and $0.75 \mu \mathrm{L}$ spots were applied on DPH matrix-free areas of the glass slide in the vicinity of the section to be imaged. After the calibration spots dried, glass slides were dehydrated in a vacuum chamber for $5-10 \mathrm{~min}$.

Matrix Sublimation. The sublimation protocol used by this research group was previously reported. ${ }^{39}$ The amount of matrix, temperature and duration were reoptimized for DPH: $250 \mathrm{mg} \mathrm{DPH}$ at $200{ }^{\circ} \mathrm{C}$ for $3.0 \mathrm{~min}$. These conditions produced a uniform layer of matrix crystals on the entire surface of ITO glass. The sublimation time was also optimized by examining the total ion counts (TIC) between the $\mathrm{m} / \mathrm{z}$ range of 600 to 1000 from mouse brain tissue covered with various thicknesses of DPH. The TIC recorded were 0.005, 0.010, 0.025, 0.020 and 0.018, respectively from DPH coverages of 70, 85, 90, 120, and $150 \mu \mathrm{g} \mathrm{per} \mathrm{cm}^{2}$. Hence, the coverage of $90 \mu \mathrm{g}$ per $\mathrm{cm}^{2}$, resulted from a sublimation time of $3.0 \mathrm{~min}$ was deemed optimal. With the naked eyes, an optimal coating of matrix was thick enough to appear as a translucent coating, yet sufficiently thin to allow the shape and details of brain to remain visible.

MALDI MS, MSI and MS/MS. MS data were obtained using a SCIEX 5800 MALDI TOF-TOF mass spectrometer. The instrument is equipped with a $349 \mathrm{~nm} \mathrm{Nd:YLF} \mathrm{"OptiBeam} \mathrm{On-Axis"} \mathrm{laser.} \mathrm{The} \mathrm{laser}$ pulse rate used was $400 \mathrm{~Hz}$. Data acquisition and processing of profiling data were respectively done using a TOF-TOF Series Explorer and Data Explorer (SCIEX). ITO-coated glass slides with tissue sample sections were mounted onto a MALDI plate adapter and loaded into the mass spectrometer. Following the external calibration at $\pm 50 \mathrm{ppm}$ mass tolerance, images were acquired in reflectron negative ion mode, with 70-80 $\mu \mathrm{m}$ laser steps. The laser energy was optimized in MSI experiments to procedure sufficiently high signal intensities for fatty acids and minimal the fragmentation of lipids. For the results presented in this paper, the laser intensities used were typically around 2600 (arbitrary unit). However, this number will vary with the age of the laser. The detector multiplier was set at $1.89 \mathrm{~V}$ for MS mode. A mass range between $\mathrm{m} / \mathrm{z}$ of $200-1000$ was acquired. All experiments were repeated at least three times to ensure reproducibility.

Negative ion MS/MS mode experiments were conducted with 10 to $20 \%$ higher laser energy compared to the MS mode, in order to promote fragmentation of the analytes of interest for structural identification. The detector multiplier voltage was also maximized at $2.25 \mathrm{kV}$ for MS/MS mode. Following the external calibration at $\pm 50 \mathrm{ppm}$ tolerance, the precursor ion was isolated within an $\mathrm{m} / \mathrm{z}$ window of $\pm 0.2 \mathrm{Da}$ and fragmented in a postsource decay mechanism. Besides the MS/MS experiments, lipid characterization was 
performed by comparing accurate mass measurements with the LIPID MAPS prediction tool (http://www.lipidmaps.org/tools/index.html). Certain peak assignments were based on work reported by Wang et al. ${ }^{40}$

Processing of Molecular Image Data. MS imaging data were visualized using MSiReader (Version 0.06). ${ }^{41}$ The images presented in this paper have not been smoothed, normalized, or preprocessed. All images were normalized for visualization of expression; that is, the same shades and colors from different images do not reflect the same absolute signal intensities. 


\section{Results and Discussion}

\section{DPH as a Matrix for MALDI MS}

DPH has a strong absorption in the region between 330 and $380 \mathrm{~nm}$, and it is a common fluorescence probe for studying the membrane cell due to its high affinity to acyl chains. ${ }^{34}$ Such characteristics of DPH prompted us to investigate its application as a MALDI matrix for the detection of fatty acids and phospholipids. However, the strong UV-absorptivity of DPH may also give rise to the formation of abundant metastable matrix cluster ions during the laser desorption/ionization process, resulting in "noisy" backgrounds. Therefore, our initial experiment was to record the DPH matrix background MALDI MS signals in both positive and negative ion modes. The spectra are respectively presented as Figure 1 a and b. In the positive ion mode, the background spectrum is extremely clean and primarily dominated by the $\mathrm{DPH}$ radical ion $[\mathrm{M} \cdot]^{+}$at $\mathrm{m} / \mathrm{z}$ of 232.1 (Figure 1a). In negative ion mode, the DPH background spectrum is more complex (Figure 1b). The most intense peak is from the deprotonated DPH ions, $[\mathrm{M}-\mathrm{H}]^{-}$, at $\mathrm{m} / \mathrm{z}$ of 231.2. The peak at $\mathrm{m} / \mathrm{z} 233.2$ is also intense, which corresponds to radical ion $\left[\mathrm{M}+\mathrm{H}^{\bullet}\right]^{-}$. The additional peaks with $\mathrm{m} / \mathrm{z}$ larger than $\mathrm{m} / \mathrm{z} 233.2$ were not identified, but were speculated to be impurities, clusters, or adducts of DPH.
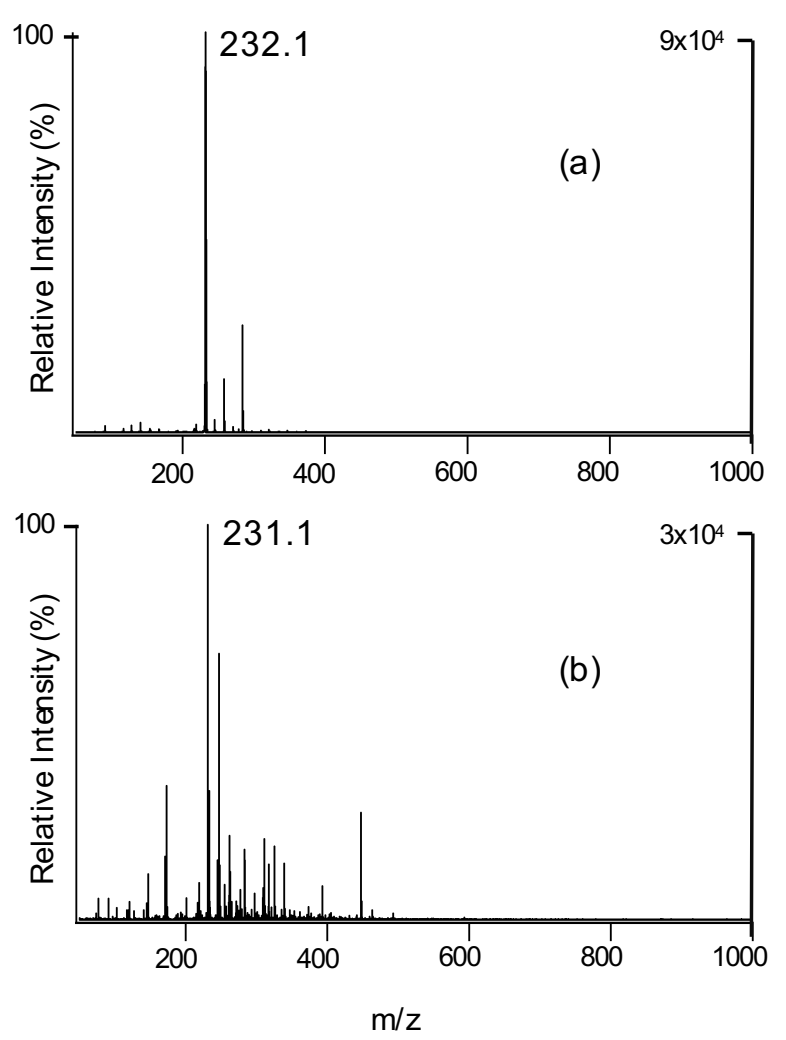

Figure 1. MALDI-TOF mass spectra of DPH matrix background in positive (a) and negative (b) ion modes. 
To determine the effectiveness of DPH as a MALDI matrix for fatty acids, three fatty acid standards, hexanoic, palmitic and arachidic acids, were used as test analytes. As shown in Figures $2 \mathrm{a}$ and $\mathrm{b}$, the expected deprotonated molecular ions of FA(16:0 and 20:0), $[\mathrm{M}-\mathrm{H}]^{-}$, were observed as the most intense peaks at $\mathrm{m} / \mathrm{z}$ of 255.2 and 311.3 respectively. The DPH matrix signals were largely undetected, including the deprotonated DPH ions (m/z of 231.2) previously observed in Figure $1 \mathrm{~b}$. This suppression of matrix background signals in the presence of analyte signals has been reported and extensively studied as matrix suppression effect (MSE), in both positive ${ }^{8}$ and negative ion modes ${ }^{42}$ with other common MALDI matrices. More discussion on this topic will be presented in the following section.

It is noteworthy that we were unable to obtain any signals of the shortest fatty acid tested, hexanoic acid. We hypothesize that the six-carbon acyl chain was too short to favorably interact with DPH for optimal analyte-matrix cocrystallization. DPH is commonly used for studying membrane bilayers consisting of lipids with chain lengths of 12 or more carbons. Others had also reported that DPH could not detect the presence of octanoic acid (8:0) in the membrane. ${ }^{43}$ Importantly, we also performed a comparison of DPH with other commonly used matrices, using palmitic and arachidic acids as analytes. Higher signal intensities were observed with DPH for both fatty acids (Supporting Information, Figure S-1).
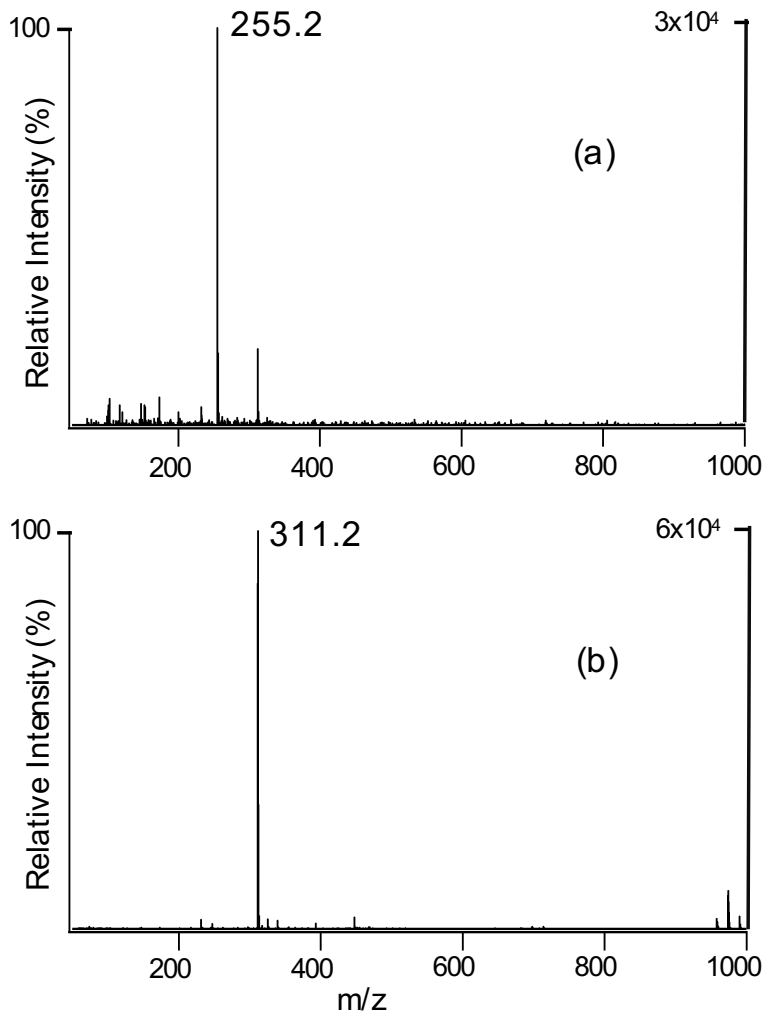

Figure 2. Negative ion mode MALDI mass spectra of (a) palmitic acid and (b) arachidic acid using DPH as a matrix. 
Next, we applied DPH for the MALDI MS detection of phospholipid standards. Table 1 lists the positive ions, mainly as sodiated and/or protonated ions, and in negative ion mode, as their deprotonated ions [M$\mathrm{H}]^{-}$, and DLPC as the $\left[\mathrm{M}-\mathrm{CH}_{3}\right]^{-}$ion. The latter has been reported previously in phosphatidylcholine detection using 9-AA as a matrix. ${ }^{44}$ While DPH facilitated the MALDI MS detection of all phospholipid standards examined, the observed signal intensities were generally higher when analyzed in negative ion mode (Supporting Information, Figure S-2). DPH is a neutral molecule with multiple double bonds, we hypothesize that it behaves more favorably as a proton acceptor than a proton donor, and thus, analyte ionization occurs mainly through the abstraction of protons. Hence, we focused our remaining work in negative ion mode, and only the negative-ion MALDI-TOF mass spectra of these phospholipid standards are presented (Supporting Information, Figure S-3). We suspect that the signal intensities of phospholipids can be enhanced in the positive ion mode by addition of salt ions, but such investigation is out of the scope of this paper.

Table 1. Positive and Negative Ions Observed in MALDI-MS for LPA, LPC, DMPA, DLPC, DSPE, DSPG, and DOPS Standards Using DPH as a Matrix.

\begin{tabular}{|c|c|c|c|c|}
\hline Analyte & $\begin{array}{l}\text { Positive Ions } \\
\text { m/z observed }\end{array}$ & Ions & $\begin{array}{l}\text { Negative Ions } \\
\text { m/z observed }\end{array}$ & Ions \\
\hline LPA & $\begin{array}{l}433.2 \\
455.2 \\
477.2\end{array}$ & $\begin{array}{c}{[\mathrm{M}+\mathrm{Na}]^{+},} \\
{[\mathrm{M}-\mathrm{H}+2 \mathrm{Na}]^{+},} \\
{[\mathrm{M}-2 \mathrm{H}+3 \mathrm{Na}]^{+}}\end{array}$ & 409.2 & {$[\mathrm{M}-\mathrm{H}]^{-}$} \\
\hline LPC & 480.3 & {$[\mathrm{M}+\mathrm{H}]^{+}$} & 464.3 & {$\left[\mathrm{M}-\mathrm{CH}_{3}\right]^{-}$} \\
\hline DMPA & $\begin{array}{l}615.4 \\
637.4\end{array}$ & $\begin{array}{c}{[\mathrm{M}+\mathrm{Na}]^{+}} \\
{[\mathrm{M}-\mathrm{H}+2 \mathrm{Na}]^{+}}\end{array}$ & 591.4 & {$[\mathrm{M}-\mathrm{H}]^{-}$} \\
\hline DLPC & $\begin{array}{l}622.4, \\
644.4 \\
666.4\end{array}$ & $\begin{array}{c}{[\mathrm{M}+\mathrm{H}]^{+}} \\
{[\mathrm{M}+\mathrm{Na}]^{+}} \\
{[\mathrm{M}-\mathrm{H}+2 \mathrm{Na}]^{+}}\end{array}$ & 606.4 & {$\left[\mathrm{M}-\mathrm{CH}_{3}\right]^{-}$} \\
\hline DSPE & $\begin{array}{l}770.6 \\
792.6\end{array}$ & $\begin{array}{c}{[\mathrm{M}+\mathrm{Na}]^{+},} \\
{[\mathrm{M}-\mathrm{H}+2 \mathrm{Na}]^{+}}\end{array}$ & 746.6 & {$[\mathrm{M}-\mathrm{H}]^{-}$} \\
\hline DSPG & $\begin{array}{l}823.5, \\
839.5 \\
855.5\end{array}$ & $\begin{array}{c}{[\mathrm{M}-\mathrm{H}+2 \mathrm{Na}]^{+},} \\
{[\mathrm{M}-\mathrm{H}+\mathrm{Na}+\mathrm{K}]^{+},} \\
{[\mathrm{M}-\mathrm{H}+2 \mathrm{~K}]^{+}}\end{array}$ & 777.6 & {$[\mathrm{M}-\mathrm{H}]^{-}$} \\
\hline DOPS & $\begin{array}{l}810.5 \\
832.5 \\
854.5\end{array}$ & $\begin{array}{c}{[\mathrm{M}+\mathrm{Na}]^{+}} \\
{[\mathrm{M}-\mathrm{H}+2 \mathrm{Na}]^{+}} \\
{[\mathrm{M}-2 \mathrm{H}+3 \mathrm{Na}]^{+}}\end{array}$ & $\begin{array}{l}786.5 \\
808.5\end{array}$ & $\begin{array}{c}{[\mathrm{M}-\mathrm{H}]^{-},} \\
{[\mathrm{M}+\mathrm{Na}-2 \mathrm{H}]^{-}}\end{array}$ \\
\hline
\end{tabular}


While intense MALDI MS signals of phospholipids were observed in the negative ion mode using DPH, we wanted to investigate how well DPH performs relative to other common matrices suitable for phospholipids. A comparison was therefore performed on mixture of the four phospholipids, DMPA, DSPE, DSPG and DOPS, using four matrices, DHB, 9-AA, DAN and DPH. The resulting MALDI mass negative ion mode spectra and average signal-to-noise ratios $(\mathrm{S} / \mathrm{N})$ are presented in Figure S-4 (Supporting Information) and Table 2. Interestingly, none of the four matrices appeared to provide a universal advantage in promoting the $\mathrm{S} / \mathrm{N}$ of these phospholipids. Instead, differences in selectivity or bias were observed. Specifically, DMPA had the highest S/N with 9-AA, DSPE was strongest with DAN, DSPG was best detected using DPH, and DOPS was most favorable with DHB. The optimal matrices for these four phospholipids were underlined in Table 2. The result is not surprising and is likely related to different degree of interaction for each matrix toward the different lipid molecules. While there was not a universal enhancement in using DPH as a matrix for phospholipids, the main advantage of DPH lies in the superior detection of fatty acids. Importantly, one should regard DPH as desirable for its ability to allow simultaneous detection of both fatty acids $(\mathrm{m} / \mathrm{z} \sim 200-350)$ as well as the larger phospholipids $(\mathrm{m} / \mathrm{z} \sim 600$ - 1000), which will be fully demonstrated in the imaging MS experiments in a later section. For the same reason, we also did not compare the sensitivity of phospholipids using DPH in negative ion mode with that using other matrices in positive ion mode.

Table 2: Average Signal-to-Noise Ratios and Standard Deviations (SD) from Triplicate MALDI MS Analyses of DMPA, DSPE, DSPG. and DOPS Using 9-AA, DAN, DHB, and DPH as Matrices.

\begin{tabular}{|c|c|c|c|c|}
\hline Matrix & $\begin{array}{c}\text { DMPA (S/N) } \\
\text { m/z } 591.4\end{array}$ & $\begin{array}{c}\text { DSPE }(\mathrm{S} / \mathrm{N}) \\
\mathrm{m} / \mathrm{z} 746.6\end{array}$ & $\begin{array}{c}\text { DSPG (S/N) } \\
\text { m/z } 777.6\end{array}$ & $\begin{array}{c}\text { DOPS }(\mathrm{S} / \mathrm{N}) \\
\mathrm{m} / \mathrm{z} 786.5\end{array}$ \\
\hline 9-AA & 2958, SD 213 & 733, SD 60 & 4076, SD 137 & 3311, SD 97 \\
\hline DAN & 2008, SD 114 & 5034, SD 46 & 6810, SD 239 & 2422, SD 87 \\
\hline DHB & 53, SD 5 & 632, SD 13 & 2090, SD 43 & $\underline{4159}, \mathrm{SD} 66$ \\
\hline $\mathrm{DPH}$ & 1326, SD 24 & 2066, SD 37 & 8940, SD 214 & 1043, SD 34 \\
\hline
\end{tabular}

\section{Insight on the Cocrystallization and Matrix Suppression Effect (MSE):}

McCombie and Knochenmuss proved the utility of MSE for the detection of low molecular weight compounds in MALDI-MS, by optimizing the laser fluence and matrix-to-analyte molar ratios (m/a ratio). ${ }^{45}$ It has been also shown that analyte-matrix proximity is an important requirement to produce the MSE. ${ }^{8,42}$ 
We aimed to explore this phenomenon and the influence of solvent on analyte signal using DPH. As a fluorescence probe, typically used at a concentration range of $10^{-7}$ to $10^{-6} \mathrm{M}$, DPH can be dispersed in aqueous media to form microaggregates, which are practically nonfluorescent. When such an aqueous dispersion is mixed with lipids, the DPH molecules integrate and interact with the hydrophobic domain of the lipids, and exhibit a dramatic increase in fluorescence. ${ }^{37}$ For our MALDI MS experiments, we hypothesized that optimal cocrystallization between DPH and analyte can be achieved by promoting proximity or intimate interactions. One way to achieve this is to use water in a similar fashion as in the fluorescence experiments aforementioned. Specifically, the addition of water to a nonpolar environment will drive the nonpolar DPH molecules toward the hydrophobic region of the analytes, such as fatty acids or phospholipids in this case. To illustrate this effect, we performed MALDI MS analyses on a mixture of three analytes, palmitic acid, arachidic acid and lyso-phosphatidic acid, using DPH as the matrix, with and without the presence of water. The results are presented in Supporting Information (Figure S-5), where weaker matrix background signals and slightly more intense analyte signals were observed in the presence of water. This discovery supports our hypothesis of tighter interactions between DPH and the acyl chains of lipids and fatty acids being essential to promoting efficient energy transfer during the MALDI process.

\section{DPH Matrix for MS Imaging}

After DPH has been ascertained as a suitable MALDI matrix for fatty acids and phospholipids, its application in mass spectrometry imaging will be illustrated next. DPH is soluble in only selective solvent such as THF or acetone. While the DPH solution in either THF or acetone works well for deposition with micropipettes or automated sprayer, we are most interested in the application of DPH via sublimation, which eliminates the use of solvents and thus minimizes the delocalization of analytes on the tissue, ${ }^{46}$ resulting in MSI data of high spatial resolution and reproducibility. ${ }^{47}$ However, for a matrix to be a useful in MALDI MS imaging, it must remain relatively nonvolatile under vacuum. This was a limitation of DMAN, which sublimes significantly at room temperature under vacuum, making it unsuitable for MSI. To demonstrate the low volatility of DPH under vacuum, we loaded glass slides with DPH-coated tissue sections into the mass spectrometer, and allowed them to sit at various time periods of $0,6,12$, and $24 \mathrm{~h}$ under vacuum $\left(10^{-7}\right.$ Torr), prior to acquiring the mass spectra (Supporting Information, Figure S-6). A total of 70 main lipid peaks were detected and identified, no significant changes in overall signal intensities were found. These results demonstrate the low volatility of DPH under high vacuum conditions for up to the 24 $\mathrm{h}$ tested.

Due to the high content and diversity of lipids in the central nervous system, brain tissues are excellent samples for this work. Figure 3a presents the MALDI-TOF mass spectrum in the negative-ion mode acquired from a coronal cerebellum mouse brain tissue section between the $\mathrm{m} / \mathrm{z}$ range of $200-1000$. In 
general, signals from a diverse variety of lipids were observed. In this work, we are particularly interested in the detection of fatty acids, since the existing common MALDI matrices are not optimal for such an application. To illustrate the detection of fatty acids from brain tissue using DPH, Figure $3 \mathrm{~b}$ shows the mass spectrum focusing on the $\mathrm{m} / \mathrm{z}$ range of $200-350 \mathrm{Da}$. The peaks at $\mathrm{m} / \mathrm{z} 253.2,255.2,279.2,281.2,283.3$, 303.2, 309.2, 327.3, 331.2, respectively, corresponding to palmitoleic acid (16:1), palmitic acid (16:0), linoleic acid (18:2), oleic acid (18:1), stearic acid (18:0), arachidonic acid (20:4), gadoleic acid (20:1), docoshexaenoic acid (22:6), and docosatetraenoic acid (22:4) were observed. Moreover, we noticed an optimal matrix suppression effect in this case where nearly zero matrix background ions were observed.
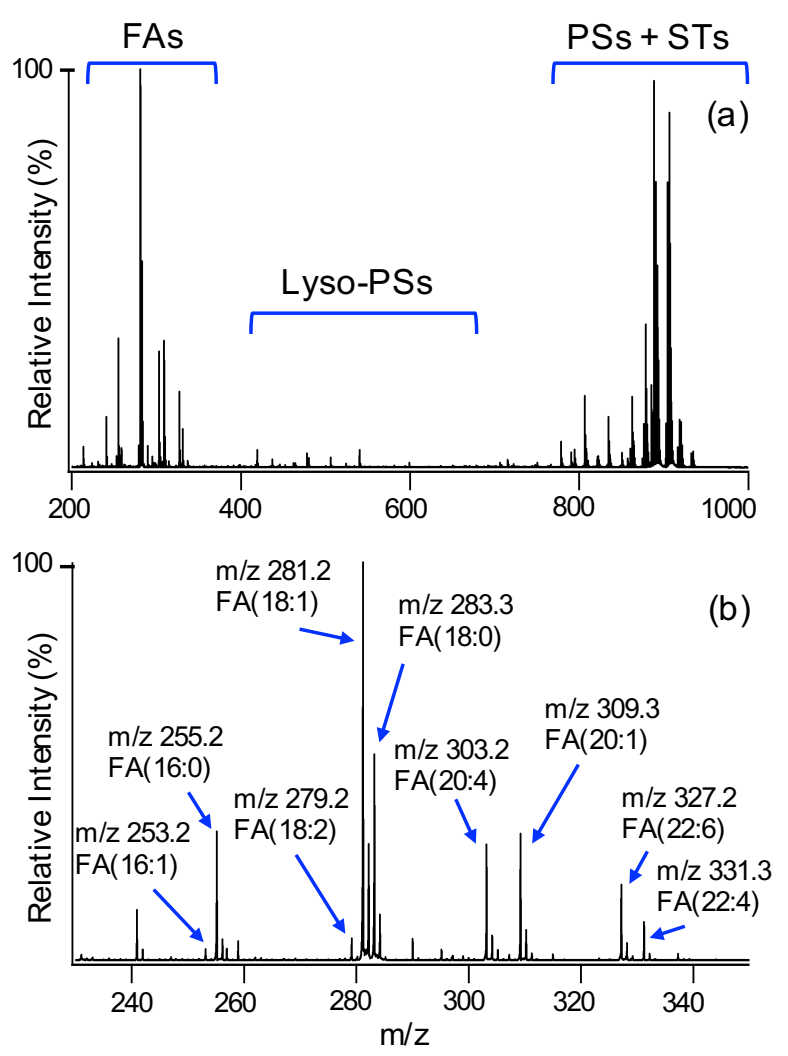

Figure 3: Negative ion mode MALDI-TOF mass spectra acquired from coronal cerebellum mouse brain section coated with DPH via sublimation, showing the full $\mathrm{m} / \mathrm{z}$ range of $200-1000$ (a) and the $\mathrm{m} / \mathrm{z}$ range of 230 - 350 focusing on fatty acids (b).

The intense $\mathrm{S} / \mathrm{N}$ observed in Figure 3 allows us to obtain MS images of various fatty acids and lipids at extremely high quality. The ion images of selected fatty acids and phospholipids are presented in Figure 4, which show the structure of coronal cerebellum mouse brain section with distinguishable localization of ion distribution in different compartments such as cerebellar lobuls, middle cerebellar penducle, and fourth ventricle. 


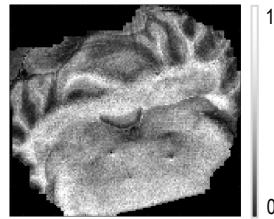

$\mathrm{m} / \mathrm{z} \mathbf{2 8 1 . 2}$

$\mathrm{FA}(18: 1)$

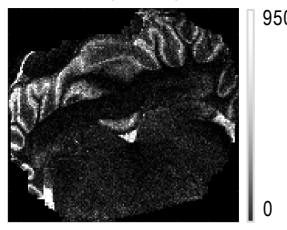

$\mathrm{m} / \mathrm{z} 599.3$

lyso-PI(18:0)

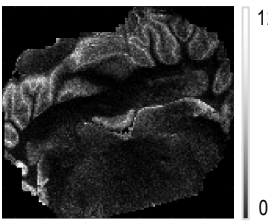

$\mathrm{m} / \mathrm{z} \mathbf{8 5 7 . 5}$

$\mathrm{PI}(36: 4)$

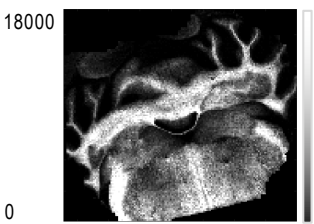

$\mathrm{m} / \mathrm{z} 309.3$

$\mathrm{FA}(20: 1)$

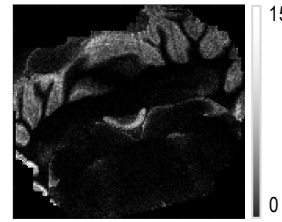

$\mathrm{m} / \mathrm{z} 715.6$

$\mathrm{PA}-\mathrm{O}(38: 1)$

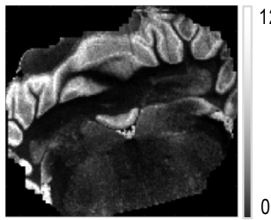

$\mathrm{m} / \mathrm{z} 885.5$

$\mathrm{PI}(38: 4)$

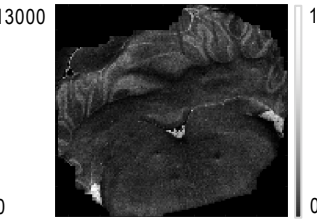

$\mathrm{m} / \mathrm{z} \mathbf{3 0 3 . 2}$

$\mathrm{FA}(20: 4)$

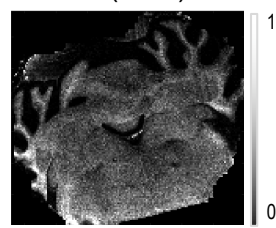

$\mathrm{m} / \mathrm{z} 794.6$

$\operatorname{PE}(40: 4)$

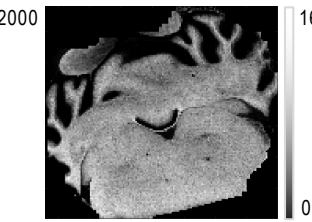

$\mathrm{m} / \mathrm{z} \mathbf{8 8 8 . 6}$

ST(24:1)

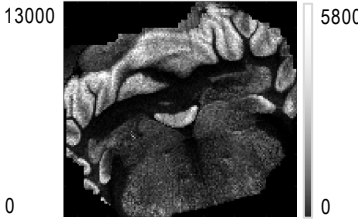

$\mathrm{m} / \mathrm{z} 327.2$

$\mathrm{FA}(22: 6)$

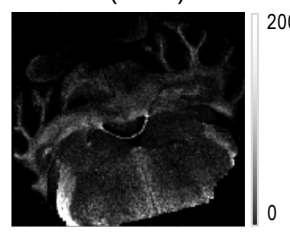

$\mathrm{m} / \mathrm{z} \mathbf{8 2 1 . 5}$

PG(40:6)

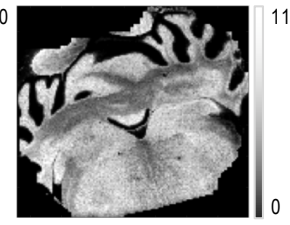

$\mathrm{m} / \mathrm{z} 906.6$

$\mathrm{ST}(24: 0(\mathrm{OH}))$

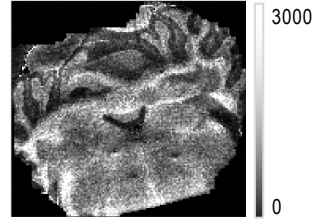

$\mathrm{m} / \mathrm{z} 331.3$

$\mathrm{FA}(22: 4)$

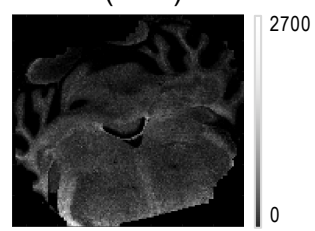

$\mathrm{m} / \mathrm{z} \mathbf{8 3 4 . 5}$

PS(40:6)

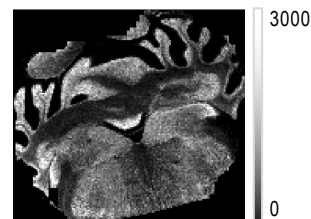

$\mathrm{m} / \mathrm{z} \mathbf{9 2 0 . 7}$

$\mathrm{PE}(46: 2) \quad 2 \mathrm{~mm}$

Figure 4. MALDI-MS images of coronal cerebellum mouse brain section (Interaural -1.08, Bregma -10.08 , Paxinos and Watson Atlas) coated with DPH by sublimation acquired in negative-ion mode, spatial resolution at $80 \mu \mathrm{m}$.

In Figure 5, the MALDI-MS images of coronal hippocampus rat brain section indicate that many sulfatides (STs) species were detected: $\mathrm{m} / \mathrm{z} 778.5,806.5,878.6,862.5,888.6,904.6$, and 906.6. They were found to be more abundant in the white matter (corpus callosum) than in the hippocampus and cortex. This characteristic distribution is in agreement with previously published work. ${ }^{48}$ In addition, we were able to detect small peaks at 822.5, 834.6, 850.6, 916.7, 918.7, 932.6, and 934.7, and they all exhibited similar distribution patterns (data not shown). 


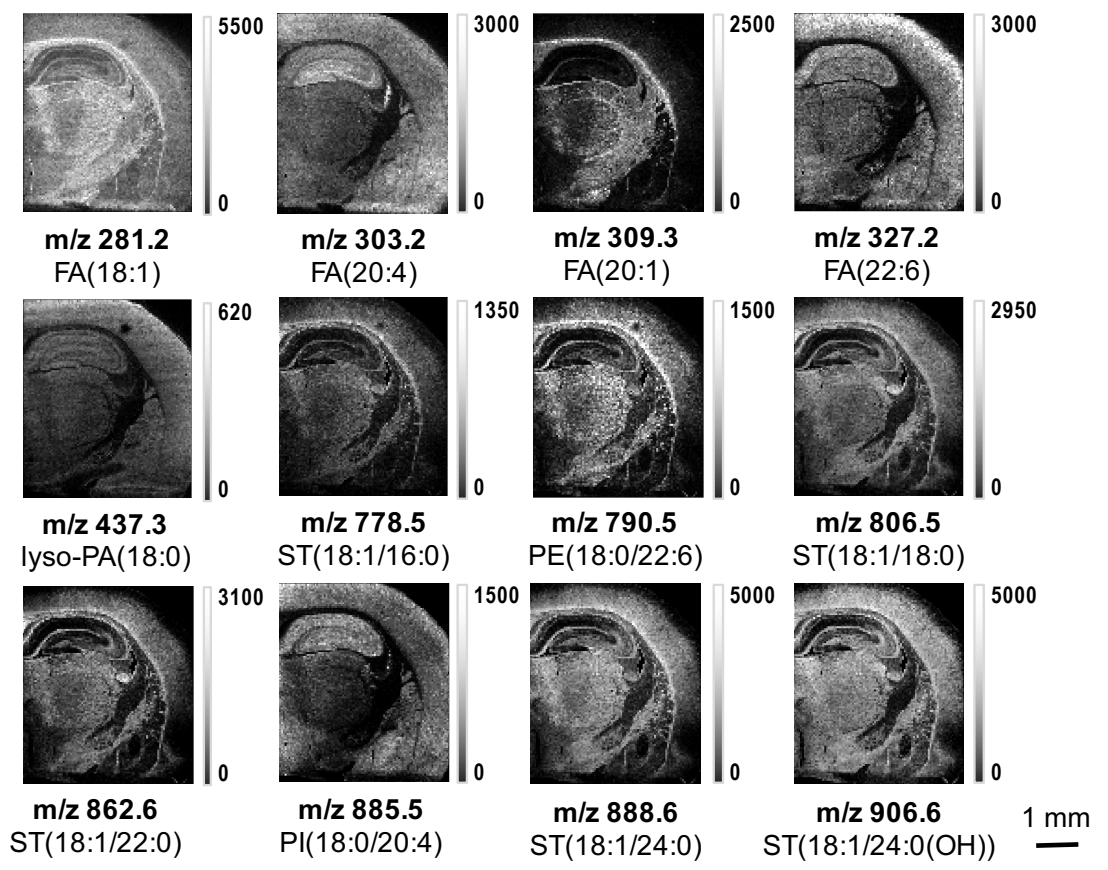

Figure 5: MALDI MS images of coronal hippocampus section of rat brain (Interaural 5.64, Bregma -3.36, Paxinos and Watson Atlas) acquired in negative-ion mode using sublimated DPH as matrix, spatial resolution $70 \mu \mathrm{m}$.

As demonstrated in Figures 4 and 5 and the additional Figure S-7 (Supporting Information) showing two serial sagittal sections of the same mouse brain, MSI using DPH as the matrix provided a significant amount of information on the distribution and abundance of a number of fatty acids and lipids in different anatomical regions including cortex, corpus callosum, hippocampus, caudate putamen (striatum), and lateral ventricle.

Despite the high-quality images shown in the above figures, it is acknowledged that the fatty acid signals measured by mass spectrometry may come from endogenous free fatty acids as well as fatty acids dissociated from phospholipids during ionization. This is a general limitation of mass spectrometry and is not directly related or affected by the choice of MALDI matrix. Others have attempted to differentiate between the free and dissociated forms of fatty acids by chemically labeling the free fatty acids with esterification. ${ }^{30}$ Unfortunately, it was unsuccessful due to the hydrolysis of phospholipids during the derivatization process.

To gauge the level of phospholipid fragmentation in our work with DPH, we first performed MALDI MS measurements on DMPA standards at various laser intensities, since high laser energies is known to promote fragmentation of analytes. ${ }^{49-51}$ The results in Figure S-8 (Supporting Information) illustrate that 
myristic acid resulted from fragmentation of DMPA was not detected at the lowest laser energy (Figure S8a). With higher laser energies, increasing intensities of myristic acid and decreasing intensities of intact DMPA were observed. In the worst-case scenario, at the highest laser energy examined, all of the DMPA were fragmented (Figure S-8e). Based on this set of data, the use of low laser energy is preferred for the suppression of phospholipid fragmentation, although it needs to be sufficiently high to induce analyte ionization.

In the next experiment, spots of DSPG (m/z 777.6) and DSPE (m/z 746.6) standards were deposited on mouse brain tissue. After DPH sublimation, the degree of fragmentation was determined at three laser intensities, 2600, 2700, and 2800. The strongest fragment peaks resulted from these lipids were at $\mathrm{m} / \mathrm{z} 493.3$ and 462.6, respectively, which corresponded to the loss of one fatty acid chain (stearic acid, C18). The results are presented in Supporting Information, Figure S-9a, and it confirms that the level of lipid fragmentation increased with higher laser energies. In Figure S-9b, we plotted the signals of deprotonated stearic acid (m/z 283.3) on the spiked spots of DSPG and DSPE, which likely resulted from the fragmentation of DSPG and DSPE. For comparison, we showed intensities of the "endogenous" 283.3 peak from the tissue (outside of the spiked spots), which increased with increasing laser energies. In addition, we displayed the signals from two larger lipids, m/z 885.5 from phosphoinositol (18:0/20:4) and m/z 888.6 from sulfatide (18:1/24:1) detected from the tissue, to illustrate the decreasing signals of intact phospholipids at higher laser intensities. The data in Figure S-9 collectively illustrated that lower laser energy, 2600 in this case, is favorable in minimizing the fragmentation of phospholipids. Laser energies below 2600 was also tested, but poor sensitivity was generally resulted. Consequently, all of the tissue imaging data presented in this paper was collected at a laser energy of 2600. While we are unable to claim that the fatty acids detected at this low laser energy were all endogenous free fatty acids, we are confident that most of them were indeed free fatty acids. Importantly, one may also collect parallel MS images at high laser energy; such as 2800 in this case. Based on changes in fatty acid distribution between low and high laser energy, one may be able to differentiate the endogenous fatty acid from fragments of phospholipids.

On-Tissue Structural Identification of Lipids by Tandem Mass Spectrometry. As the final section of this paper, we investigated the performance of DPH as a matrix in facilitating on-tissue tandem MS analysis of lipids. We began our studies by evaluating the fragmentation patterns of lipid standards under MALDI tandem MS using DPH as a matrix. As demonstrated in Figure S-10 (Supporting Information), DMPA and DSPG produced very clean spectra with easily identifiable fragments. Next, we conducted tandem MS on the intense lipid signals detected from tissue sections, with DPH applied via sublimation. Thanks to the highly sensitive detection of lipids from the tissue, tandem MS characterization was successfully achieved 
on-tissue. As demonstrated in Figure 6, the precursor ion with $\mathrm{m} / \mathrm{z} 885.5$ was fragmented into product ions with $\mathrm{m} / \mathrm{z} 439.2,419.2,303.2,283.3,259.2$, and 241.0. The fragment ion of $\mathrm{m} / \mathrm{z} 439.2$ corresponds to a molecule generated by a neutral loss of arachidonic acid (20:4, $304 \mathrm{Da})$ and inositol phosphate (162 Da; $\mathrm{C}_{6} \mathrm{H}_{10} \mathrm{O}_{5}$ ). Another fragment ion of $\mathrm{m} / \mathrm{z} 419.3$ came from the neutral loss of stearic acid (18:0, $\left.284 \mathrm{Da}\right)$ and inositol from $[\mathrm{M}-\mathrm{H}]^{-}$of precursor ion. The two peaks of $\mathrm{m} / \mathrm{z} 283.3$ and 303.2 correspond to the $[\mathrm{M}-\mathrm{H}]^{-}$ions of stearic and arachidonic acid, respectively. Fragment ion m/z 259 is inositol phosphate ion, and m/z 241 is the headgroup of inositol phosphate ion with loss of $\mathrm{H}_{2} \mathrm{O}$. These results collectively confirm the parent molecule of $\mathrm{m} / \mathrm{z} 885.5$ as phosphatidylinositol (PI 18:0/20:4). Interestingly, one may notice the fragment ion peak intensity of arachidonate derived from the sn-2 position being significantly higher than that of stearate derived from the sn-1 position. The same was observed from the two peaks of $\mathrm{m} / \mathrm{z} 439$ and 419 that correspond, respectively, to the complementary fragment of each fatty acids.

Similar to PI, fragmentation of other phospholipid classes such as PE, PG, and PA also yields specific diagnostic fragment peaks corresponding to their specific fatty acids, and, thus, allowing the identification of isobaric lipid molecules. Figure S-11 (Supporting Information) shows the MS/MS spectrum of the precursor ion of $\mathrm{m} / \mathrm{z}$ 747.5, which represents two isobaric molecules PA (18:0/22:6) and PG (16:0/18:1). Through the above examples, we demonstrated that the use of DPH as a MALDI matrix was highly effective in facilitating tandem MS analysis that led to the definitive structural assignments of lipids, specifically through the multiple informative fragment ions including the fatty acids fragments.

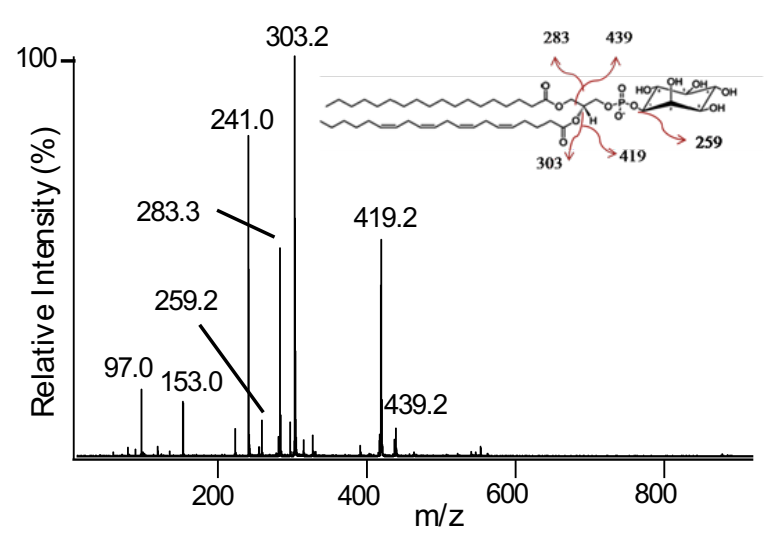

Figure 6. Tandem mass spectrum generated from the precursor ion of $\mathrm{m} / \mathrm{z} 885.5$ (PI 18:0/20:4).

Supporting Information, Table S-1, lists the relatively high intensity mass peaks observed in the negativeion MALDI-TOF-mass spectra within the $\mathrm{m} / \mathrm{z}$ range of $200-1000$, acquired from coronal cerebellum mouse. The table shows the assignment for each peak, based on both MS and tandem MS data, as described in Materials and Methods. In summary, DPH allowed the detection of 11 fatty acids, 14 lyso-phospholipids 
and lyso-sulfatide, ${ }^{52} 3$ phosphatidic acids, 16 phosphoethanolamines, 4 phosphatidylserines, 2 phosphatidylglycerols, 3 phosphatidylinositols, and 16 sulfatides. There were also many other low intensity peaks that resulted in seemingly meaningful MS images, but were not yet identified for this paper.

\section{Conclusions}

We have successfully demonstrated the application of commercially available DPH as a new MALDI matrix for the detection of fatty acids and a variety of lipids. The matrix was found to be more effective in producing intense signals in the negative ionization mode. Based on our understanding derived from this work, the hydrophobic polyene structure and its favorable interaction with the acyl chain were responsible for the ideal characteristics of DPH as a matrix. Two additional features of DPH, namely, compatibility with sublimation matrix application and low volatility in vacuum, make it an ideal matrix for MALDI MS imaging. By keeping the laser power relatively low, we were able to minimize fragmentation of phospholipids and obtain high quality MALDI MSI data in the negative ion mode. This allows the simultaneous visualization of both fatty acid and the lipid distributions from mouse and rat brain tissues, including fatty acids, lyso-phospholipid, phosphatidic acid, phosphoethanolamine, phosphatidylserine, phosphatidylglycerol, phosphatidylinositol, and sulfatides. Finally, we demonstrated the highly effective on-tissue tandem MS analysis of lipids detected from the tissue. DPH facilitated the fragmentation of lipids in a simple and easily identifiable fashion, in turn allowing the fragment information to be used for structural characterization of the precursor ions.

\section{ACKNOWLEDGMENTS}

This work was funded by the Natural Sciences and Engineering Research Council of Canada (NSERC). The financial support for H.I. was provided by the Faculty of Science at Western University through the assistance of Dr. Julie McMullin, Dr. Charmaine B. Dean, Dr. Yining Huang, and Dr. Michael Lynk, who are gratefully acknowledged. The Scholars at Risk (SAR) organization is gratefully acknowledged for offering the opportunity for H.I. to conduct the research in this project. The authors also acknowledge the help of Dr. Nina Weishaupt from Department of Anatomy and Cell Biology of Western University for her technical support in brain tissue sectioning. The authors also acknowledge the Western University MALDI MS Facility and the Schulich School of Medicine and Dentistry for their support.

Supporting Information. The Supporting Information is available free of charge. Supplementary data of additional 11 figures and 1 table, which are non-essential details that further illustrate the performance of DPH as MALDI MS matrix. 


\section{References:}

(1) Knochenmuss, R. Analyst 2006, 131, 966-986.

(2) Chaurand, P. Bioanalysis 2015, 7, 2279-2281.

(3) Knochenmuss, R. Mass spectrom. 2013, 2, S0006.

(4) Soltwisch, J.; Jaskolla, T. W.; Hillenkamp, F.; Karas, M.; Dreisewerd, K. Anal. Chem. 2012, 84, 65676576.

(5) Hankin, J. A.; Barkley, R. M.; Murphy, R. C. J. Am. Soc. Mass Spectrom. 2007, 18, 1646-1652.

(6) Horneffer, V.; Dreisewerd, K.; Lüdemann, H.-C.; Hillenkamp, F.; Läge, M.; Strupat, K. Int. J. Mass Spectrom. 1999, 185-187, 859-870.

(7) Trimpin, S.; Räder, H. J.; Müllen, K. Int. J. Mass Spectrom. 2006, 253, 13-21.

(8) Knochenmuss, R.; Dubois, F.; Dale, M.-J.; Zenobi, R. Rapid Commun. Mass Spectrom. 1996, 10, 871877.

(9) Fukuyama, Y.; Tanimura, R.; Maeda, K.; Watanabe, M.; Kawabata, S.; Iwamoto, S.; Izumi, S.; Tanaka, K. Anal. Chem. 2012, 84, 4237-4243.

(10) Stoyanovsky, D. A.; Sparvero, L. J.; Amoscato, A. A.; He, R. R.; Watkins, S.; Pitt, B. R.; Bayir, H.; Kagan, V. E. Rapid Commun. Mass Spectrom. 2014, 28, 403-412.

(11) Currie, E.; Schulze, A.; Zechner, R.; Walther, T. C.; Farese, R. V., Jr. Cell Metab. 2013, 18, 153-161.

(12) Kosicek, M.; Hecimovic, S. Int. J. Mol. Sci. 2013, 14, 1310-1322.

(13) Matsumoto, J.; Sugiura, Y.; Yuki, D.; Hayasaka, T.; Goto-Inoue, N.; Zaima, N.; Kunii, Y.; Wada, A.; Yang, Q.; Nishiura, K.; Akatsu, H.; Hori, A.; Hashizume, Y.; Yamamoto, T.; Ikemoto, K.; Setou, M.; Niwa, S. Anal. Bioanal. Chem. 2011, 400, 1933-1943.

(14) Matthan, N. R.; Ooi, E. M.; Van Horn, L.; Neuhouser, M. L.; Woodman, R.; Lichtenstein, A. H. J. Am. Heart Assoc. 2014, 3.

(15) Crowe, F. L. and 34 coauthors. Am. J. Clin. Nutr. 2008, 88, 1353-1363.

(16) Yang, L.; Lv, P.; Ai, W. P.; Li, L. N.; Shen, S. S.; Nie, H. G.; Shan, Y. B.; Bai, Y.; Huang, Y. N.; Liu, H. W. Anal. Bioanal. Chem. 2017, 409, 3211-3222.

(17) Wang, S.; Chen, X.; Luan, H.; Gao, D.; Lin, S.; Cai, Z.; Liu, J.; Liu, H.; Jiang, Y. Rapid Commun. Mass Spectrom. 2016, 30, 533-542.

(18) Norris, J. L.; Caprioli, R. M. Chem. Rev. 2013, 113, 2309-2342.

(19) Batubara, A.; Carolan, V. A.; Loadman, P. M.; Sutton, C.; Shnyder, S. D.; Clench, M. R. Rapid Commun. Mass Spectrom. 2015, 29, 1288-1296.

(20) Anderson, D. M.; Mills, D.; Spraggins, J.; Lambert, W. S.; Calkins, D. J.; Schey, K. L. Mol. Vis. 2013, 19, 581-592.

(21) Cerruti, C. D.; Benabdellah, F.; Laprevote, O.; Touboul, D.; Brunelle, A. Anal. Chem. 2012, 84, 21642171. 
(22) Thomas, A.; Charbonneau, J. L.; Fournaise, E.; Chaurand, P. Anal. Chem. 2012, 84, 2048-2054.

(23) Shroff, R.; Svatos, A. Anal. Chem. 2009, 81, 7954-7959.

(24) Shroff, R.; Svatos, A. Rapid Commun. Mass Spectrom. 2009, 23, 2380-2382.

(25) Calvano, C. D.; Zambonin, C. G.; Palmisano, F. Rapid Commun. Mass Spectrom. 2011, 25, 17571764.

(26) Jun, J. H.; Song, Z.; Liu, Z.; Nikolau, B. J.; Yeung, E. S.; Lee, Y. J. Anal. Chem. 2010, 82, 3255-3265.

(27) Hayasaka, T.; Goto-Inoue, N.; Zaima, N.; Shrivas, K.; Kashiwagi, Y.; Yamamoto, M.; Nakamoto, M.; Setou, M. J. Am. Soc. Mass Spectrom. 2010, 21, 1446-1454.

(28) Dufresne, M.; Thomas, A.; Breault-Turcot, J.; Masson, J. F.; Chaurand, P. Anal. Chem. 2013, 85, 33183324.

(29) Muller, L.; Kailas, A.; Jackson, S. N.; Roux, A.; Barbacci, D. C.; Schultz, J. A.; Balaban, C. D.; Woods, A. S. Kidney Int. 2015, 88, 186-192.

(30) Wu, Q.; Comi, T. J.; Li, B.; Rubakhin, S. S.; Sweedler, J. V. Anal. Chem. 2016, 88, 5988-5995.

(31) Wu, Q.; Chu, J. L.; Rubakhin, S. S.; Gillette, M. U.; Sweedler, J. V. Chem. Sci. 2017, 8, 3926-3938.

(32) Shinitzky, M.; Barenholz, Y. Biochimica et Biophysica Acta (BBA) - Reviews on Biomembranes 1978, $515,367-394$

(33) Kao, Y. L.; Chong, P. L.; Huang, C. H. Biophys. J. 1990, 58, 947-956.

(34) Dupuy, B.; Montagu, M. Analyst 1997, 122, 783-786.

(35) Kaiser, R. D.; London, E. Biochem. 1998, 37, 8180-8190.

(36) do Canto, A.; Robalo, J. R.; Santos, P. D.; Carvalho, A. J. P.; Ramalho, J. P. P.; Loura, L. M. S. Biochim. Biophys. Acta 2016, 1858, 2647-2661.

(37) Caudron, E.; Zhou, J. Y.; England, P.; Ollivon, M.; Prognon, P. Appl. Spectrosc. 2007, 61, 963-969.

(38) Ibrahim, H.; Kasselouri, A.; Raynal, B.; Pansu, R.; Prognon, P. J. Lumin. 2011, 131, 2528-2537.

(39) Caughlin, S.; Park, D. H.; Yeung, K. K.; Cechetto, D. F.; Whitehead, S. N. J. Vis. Exp. 2017.

(40) Wang, X.; Han, J.; Yang, J.; Pan, J.; Borchers, C. H. Chem. Sci. 2015, 6, 729-738.

(41) Robichaud, G.; Garrard, K. P.; Barry, J. A.; Muddiman, D. C. J. Am. Soc. Mass Spectrom. 2013, 24, 718-721.

(42) Knochenmuss, R.; Karbach, V.; Wiesli, U.; Breuker, K.; Zenobi, R. Rapid Commun. Mass Spectrom. 1998, 12, 529-534.

(43) Halevy, A. H.; Porat, R.; Spiegelstein, H.; Borochov, A.; Botha, L.; Whitehead, S. C. Physiol. Plant. 1996, 97, 469-474.

(44) Fuchs, B.; Bischoff, A.; Suss, R.; Teuber, K.; Schurenberg, M.; Suckau, D.; Schiller, J. Anal. Bioanal. Chem. 2009, 395, 2479-2487.

(45) McCombie, G.; Knochenmuss, R. Anal. Chem. 2004, 76, 4990-4997. 
(46) Murphy, R. C.; Hankin, J. A.; Barkley, R. M.; Zemski Berry, K. A. Biochim. Biophys. Acta 2011, $1811,970-975$.

(47) Gemperline, E.; Rawson, S.; Li, L. Anal. Chem. 2014, 86, 10030-10035.

(48) Sparvero, L. J.; Amoscato, A. A.; Dixon, C. E.; Long, J. B.; Kochanek, P. M.; Pitt, B. R.; Bayir, H.; Kagan, V. E. Chem. Phys. Lipids 2012, 165, 545-562.

(49) Asbury, G. R.; Al-Saad, K.; Siems, W. F.; Hannan, R. M.; Hill, H. H. J. Am. Soc. Mass Spectrom. 1999, 10, 983-991.

(50) Harvey, D. J.; Naven, T. J.; Kuster, B.; Bateman, R. H.; Green, M. R.; Critchley, G. Rapid Commun. Mass Spectrom. 1995, 9, 1556-1561.

(51) Benard, S.; Arnhold, J.; Lehnert, M.; Schiller, J.; Arnold, K. Chem. Phys. Lipids 1999, 100, 115-125.

(52) Kyogashima, M.; Tamiya-Koizumi, K.; Ehara, T.; Li, G.; Hu, R.; Hara, A.; Aoyama, T.; Kannagi, R. Glycobiology 2006, 16, 719-728. 